Historic, Archive Document

Do not assume content reflects current scientific knowledge, policies, or practices. 

ASPARAGUS

Columbia Mammoth White

pkt. 5 cts. 1 oz. 15 cts. 1-4 lb. 50 cts. Conovers Colossal

plit. 5 cts. 1 oz. 15 cts. $1-4 \mathrm{lb} .50 \mathrm{cts}$. DWARF BEANS

Green Pod Tender Varieties

1 lb. equals a little

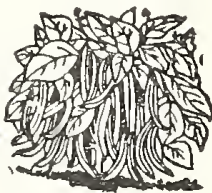

$2 \mathrm{lbs}$ equal slightly more than a quart.

5 lbs. equal about $21 / 2$ quarts.

10 lbs. equal about 5 quarts.

$15 \mathrm{lbs}$. equal one peck. $60 \mathrm{lbs}$. equal $1 \mathrm{bu}$. Improved Early Red Valentine

1 lb. 30 cts. 2 lbs. 55 cts. 5 lbs. 1.25 Bountiful Six Weeks

1 lb. 35 cts. 2 lbs. 65 cts. 5 lbs. 1.50 Burpees Stringless Green Pod

1 lb. 30 cts. 2 lbs. 55 cts. 5 lbs. 1.25 Giant Stringless Green Pod

1 lb. 30 cts. 2 lbs. 55 cts. 5 lbs. 1.25

Tennessee Green Pod

(Also called Case Knife)

1 lb. 30 cts. 2 lbs. 55 cts. 5 lbs. 1.25 Refugee or 1000 to 1

1 lb. 35 cts. 2 lbs. 65 cts. 5 lbs. 1.50 Full Measure

$1 \mathrm{lb} .35 \mathrm{cts} .2 \mathrm{lbs} .65 \mathrm{cts} .5 \mathrm{lbs} .1 .50$

\section{BRUSSELS SPROUTS}

Dwarf Improved

\section{GARDEN BEETS}

BASSANO

CROSBY'S EGYPTIAN pkt.

EDMANDS BLOOD TURNIP.... $5 c$

EARLY ECLIPSE .................... $5 c$

EXTRA EARLY EGYPTIAN ... $5 c$

EARLY BLOOD TURNIP......... $5 c$

DETROIT DARK RED

LONG SMOOTH BLOOD ........... $5 c$

SWISS CHARD (For Salad)..... $5 \mathrm{c}$

\section{STOCK BEETS}

MAMMOTH LONG RED_..... pkt.

GOLDEN TANKARD

LANES IMPERIAL。

$\begin{array}{crr}\mathrm{Oz} . & 1-41 \mathrm{~b} & 1 \mathrm{~b} \\ 10 \mathrm{c} & 30 \mathrm{c} & 90 \mathrm{c} \\ 10 \mathrm{c} & 30 \mathrm{c} & 85 \mathrm{c} \\ 10 \mathrm{c} & 30 \mathrm{c} & 85 \mathrm{c} \\ 10 \mathrm{c} & 30 \mathrm{c} & 90 \mathrm{c} \\ 10 \mathrm{c} & 30 \mathrm{c} & 85 \mathrm{c} \\ 10 \mathrm{c} & 30 \mathrm{c} & 85 \mathrm{c} \\ 10 \mathrm{c} & 30 \mathrm{c} & 85 \mathrm{c} \\ 10 \mathrm{c} & 30 \mathrm{c} & 85 \mathrm{c} \\ 10 \mathrm{c} & 30 \mathrm{c} & 95 \mathrm{c}\end{array}$

Tender GORN FIELD BEANS

Tender Green Podded Varieties

Kentucky Wonder or Old Homestead

$1 \mathrm{lb} .35$ cts. 2 lbs. 65 cts. 5 lbs. 1.50 White Kentucky Wonder Cut Short or Corn Hill

1 lb. 35 cts. 2 lbs. 65 cts. 5 lbs. 1.50 Horticultural or October

1 lb. 30 cts. 2 lbs. 55 cts. 5 lbs. 1.25

King Horticultural or Large October

1 lb. 35 cts. 2 lbs. 65 cts. 5 lbs. 1.50 Lazy Wife or White October

1 lb. 35 cts. 2 lbs. 65 cts. 5 lbs. 1.50

White Creaseback or Fat Horse

1 lb. 30 cts. 2 lbs. 55 cts. 5 lbs. 1.25

POLE WAX BEANS

Golden Cluster Wax

1 lb. 35 cts. 2 lbs. 65 cts. 5 lbs. 1.50

Kentucky Wonder Wax

1 lb. 35 cts. 2 lbs. 65 cts. 5 lbs. 1.50 more than a pint.

pkt. 5 cts. 1 oz. 25 cts. $1-4$ lb. 80 cts.

1 lb. 35 cts. 2 lbs. 65 cts. 5 lbs. 1.50
DWARF SHELL BEANS Red Kidney

1 lb. 25 cts. 2 lbs. 45 cts, 5 lbs, 1.00 White Nary or Boston Pea Bean 1 lb. 20 cts. 2 lbs. 35 cts. 5 lbs. 75 cts. White Marrow

1 lb. 30 cts. 2 lbs. 55 cts. 5 lbs. 1.25 White Kidney

1 lb. 30 cts. 2 lbs. 55 cts. 5 lbs. 1.25

DWARF OR BUSH LIMA

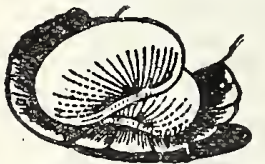

Fordhook Bush Lima Burpees Bush Lima

1 lb. 40 cts. 2 lbs. 75 cts. 5 lbs. 1.75

Hendersons Bush Lima

1 lb. 35 cts. 2 lbs. 65 cts. 5 lbs. 1.50 Woods Bush Lima

1 lb. 35 cts. 2 lbs. 65 ets. 5 lbs. 1.50

POLE LIMA BEANS

1 lb. 35 cts. 2 lbs. 65 cts. 5 lbs. 1.50 Early Leviathian

1 lb. 35 cts. 2 lbs. 65 cts. 5 lbs. 1.50

Scarlet Runner (Ornamental)

1 lb. 50 cts. 2 lbs. 95 cts. 5 lbs. 2.25
$1 \mathrm{lb} .45$ cts. 2 lbs. 85 cts. 5 lbs. 2.00

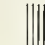

$\because$

|||

$\therefore$

$\therefore$

1

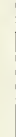

DWARF ULM SAVOY_.....10c $\quad 35 c \quad 1.30-2.50$

$\begin{array}{llll}\text { CHINESE CABBAGE } . . . . . . . .10 c & 35 \mathrm{c} & 1.30 & 2.50 \\ & & \end{array}$

CAULIFLOWER

EARLY DWARF EFFURT pkt.

EARLY SNOWBALL ...................25c

CARROTS

pkt. Oz, 1-4 lb, lb.

DANVERS HALF LONG ........... $5 \mathrm{c}$ c $10 \mathrm{c} \quad 30 \mathrm{c}$. $30 \mathrm{c}$

OX HEART

IMPROVED LONG ORANGE. 5c $10 \mathrm{c} \quad 30 \mathrm{c} \quad 90 \mathrm{c}$

$\begin{array}{rrr} & \\ \text { LARGE ROOTED } & \text { pkt. } \\ \text { COLLARDS }\end{array}$

$\begin{array}{rr} & \text { COLLARDS } \\ \text { GEORGIA GROWN } & 10 \mathrm{c}\end{array}$

CRESS WATER GARDEN

EX. CURLED (Pepper Grass) $10 \mathrm{c}$

TRUEE WATER CRESS

CELERY

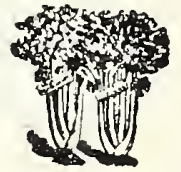

$\begin{array}{crr}\text { Oz. } & 1-4 \mathrm{lb} . & 1-2 \mathrm{lb} . \\ 25 \mathrm{c} & 90 \mathrm{c} & 1.70 \\ 25 \mathrm{c} & 90 \mathrm{c} & 1.70 \\ 25 \mathrm{c} & 95 \mathrm{c} & 1.80 \\ 25 \mathrm{c} & 95 \mathrm{c} & 1.80 \\ 30 \mathrm{c} & 1.10 & 2.10 \\ 25 \mathrm{c} & 90 \mathrm{c} & 1.70 \\ 25 \mathrm{c} & 90 \mathrm{c} & 1.70 \\ 25 \mathrm{c} & 90 \mathrm{c} & 1.70 \\ 25 \mathrm{c} & 95 \mathrm{c} & 1.80 \\ 35 \mathrm{c} & 1.30 & 2.50\end{array}$

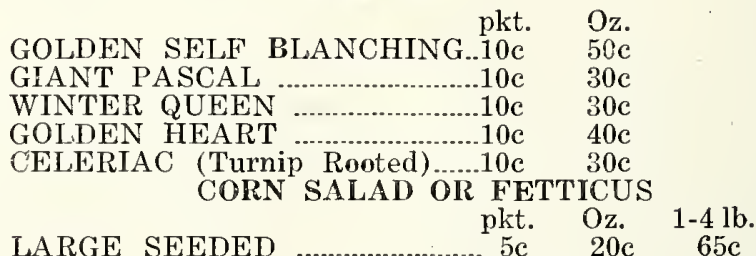

Black Mexican

1 lb. 35 cts. 2 lbs. 65 cts 5 lbs. 1.50 Stowells Evergreen

1 lb. 30 cts. 2 lbs. 55 cts. 5 lbs. 1.25 Country Gentlemen

1 lb. 30 cts. 2 lbs. 55 cts. 5 lbs. 1.25

Early Mammoth

Extra Early Adams

1 lb. 25 cts. 2 lbs. 45 cts. 5 lbs. 80 cts.

Early Adams or Burlington

1 lb. 25 cts. 2 lbs. 45 cts. 5 lbs. 80 cts. Delicious Cream

$1 \mathrm{lb}, 35 \mathrm{cts}, 2$ lbs. $65 \mathrm{cts}, 5$ lbs. 1.50 Truckers Favorite

1 lb. $30 \mathrm{cts} .2$ lbs. 55 cts. 5 lbs. 1.25 Golden Bantam

5 lbs. 1.25

1 lb. 35 cts. 2 lbs. $65 \mathrm{cts}$.

5 lbs. 1.50
$2 \mathrm{lbs} .55 \mathrm{cts}$.

5 lbs. 1.25

5 lbs. 1.25

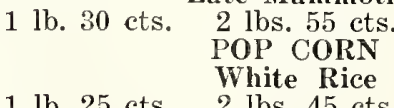

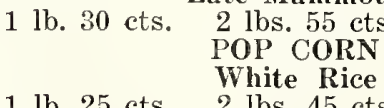

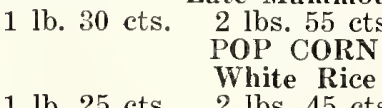

1 lb. 25 cts. 2 lbs. 45 cts.

1 lb. 25 cts. 2 lbs. 45 cts. FIELD CORN

5 lbs. 1.00

5 lbs. 1.00
1 qt. 25 cts. 1 gal. 80 cts. 1 pk. 1.15 1 bu. 4.00

Improved Golden Dent

1 qt. 25 cts. 1 gal. 90 cts. 1 pk. 1.25 1 bu. 4.25

King Philip (White Dent)

1 qt. 25 cts. 1 gal. 90 cts. 1 pk. 1.25 1 bu. 4.25

Virginia White Dent

1 qt. 25 cts. 1 gal. 80 cts. 1 pk. 1.15 1 bu. 4.00

Boone County White Dent

1 qt. 25 cts. 1 gal. 80 cts. 1 pk. 1.15 1 bu. 4.00

Hickory King (Chestnut or Eight Row)

1 qt. 30 cts. 1 gal. 1.001 pk. 1.50 1 bu. 5.50 


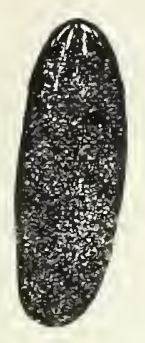

DAVIS PERFEOT

IMPROVED WHITE SPINE .... 5c

LONG GREEN

EARLY CLUSTER

EARLY FRAME

JERSEY PICKLE

JAPANESE CLIMBING …... GHERKIN

EGG PLANT

NEW YORK IMPROVED.............10

BLACK BEAUTY ENDIVE

GREEN CURLED

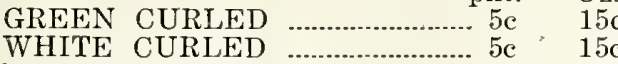
(Escarole)

\section{HERB SEEDS}

$\begin{array}{rrr} & \text { pkt. } & \text { Oz. } \\ \text { ANISE, Biennal } & 35 \mathrm{c}\end{array}$

BALM, SWEET, Perennial...........10c $35 \mathrm{c}$

CARAWAY, Perennial .................10c $35 \mathrm{c}$

DILL, Biennal ……..................... $30 \mathrm{c}$

FENNEL, SWEET, Perennial 10 $30 \mathrm{c}$

HOREHOUND, Perennial ..........10c 50c

LAVENDER, Perennial ….............10c $75 \mathrm{c}$

ROSEMARY, Perennial …..............20 c
SAGE, Annual ….......................15c

SUMMER SAVORY, Annual......15c 1.25

MARGORAM, SWEET …...........15c $95 \mathrm{c}$

THYME, Annual …........................ $\quad 2.00$ KOHL-RABI

EARLY WHITE VIENNA ......... $5 \mathrm{c} \quad \begin{gathered}\text { Oz. } \\ 30 \mathrm{c}\end{gathered}$

$\begin{array}{ccccr}\text { KALE or BORECOLE } \\ \text { pkt. } & \text { Oz. } & 1.4 \mathrm{lb} . & 1 \mathrm{~b} . \\ \text { DWARF CURLED SIBERIAN.. } & 5 \mathrm{c} & 20 \mathrm{c} & 60 \mathrm{c} & 1.75\end{array}$

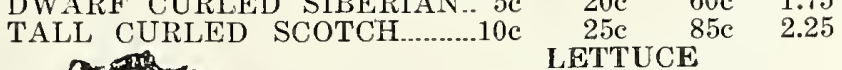

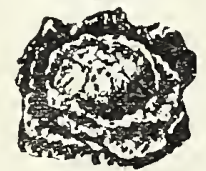

(Heading Varieties)

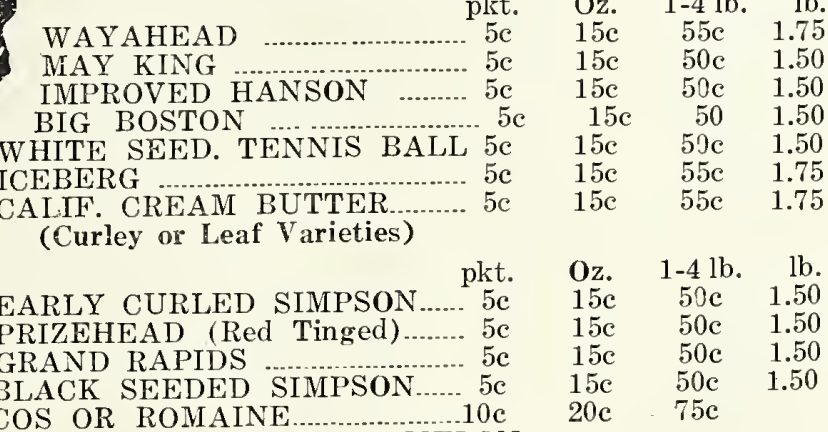

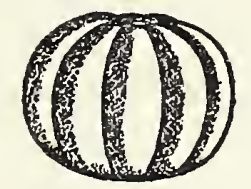

HONEY DEW

pkt. Oz. 1-4 lb.

EMERALD GEM

$20 \mathrm{c}$

EXTRA

ROCKY FORD

LATE HACKENSACK …............ 5c

WATER MELON

$15 \mathrm{c} \quad 50 \mathrm{c}$

$\begin{array}{ll}15 \mathrm{c} & 50 \mathrm{c} \\ 15 \mathrm{c} & 50 \mathrm{c}\end{array}$

$15 \mathrm{c} \quad 50 \mathrm{c}$

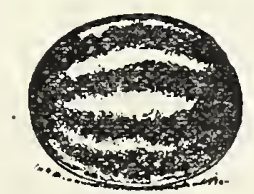

TOM WATSON

plit. Oz. 1-4 lb.

KLECKLEY SWEETS

$5 \mathrm{c}$

LONG LIGHT ICING

GEORGIA RATTLESNAKE...... $5 \mathrm{c}$

SWEET HEART

JACKSON
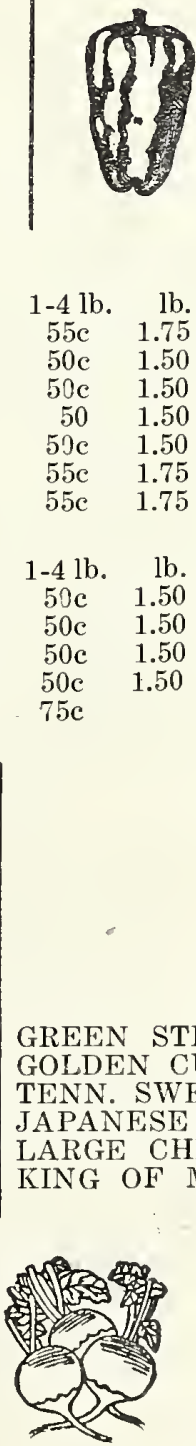

$\begin{array}{lcr}\text { Oz. } & 1-41 \mathrm{~b} . & 1 \mathrm{~b} \\ 10 \mathrm{c} & 30 \mathrm{c} & 1.00 \\ 10 \mathrm{c} & 30 \mathrm{c} & 1.00 \\ 10 \mathrm{c} & 30 \mathrm{c} & .95 \\ 10 \mathrm{c} & 30 \mathrm{c} & 1.00 \\ 10 \mathrm{c} & 30 \mathrm{c} & 1.00 \\ 10 \mathrm{c} & 30 \mathrm{c} & 1.00 \\ 10 \mathrm{c} & 35 \mathrm{c} & 1.25 \\ 15 \mathrm{c} & 50 \mathrm{c} & 1.50\end{array}$

MUST ARD

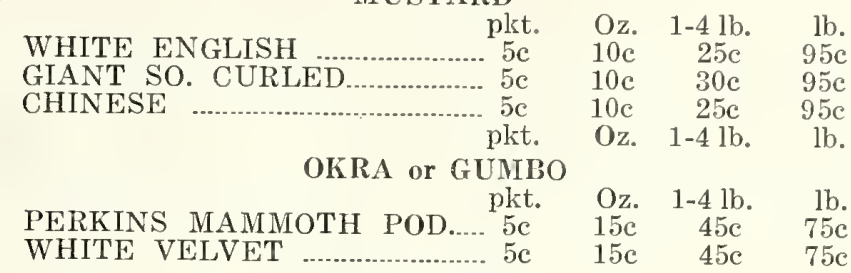

ONION SEED

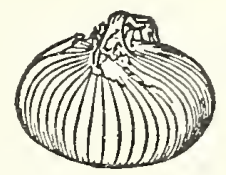

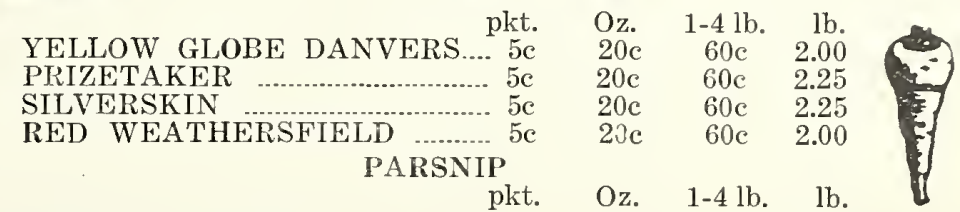
HOLLOW CROWN $\begin{array}{ll}\text { pkt. } & \mathrm{Oz} . \\ 5 \mathrm{c} & 10 \mathrm{c}\end{array}$ PARSLEY

DOUBLE CURLED MOSS CURLED

$\begin{array}{llllllll}5 \mathrm{c} & 10 \mathrm{c} & 30 \mathrm{c} & 75 \mathrm{c}\end{array}$ PEPPER

pkt. Oz. 1-4 lb. lb.

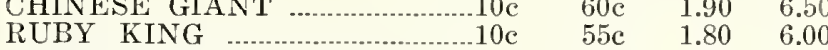
LARGE BELL or BULL NOSE.. $\quad 50 \mathrm{c} \quad 1.60 \quad 5.00$ L.ONG RED CAYANNE_............10e $45 \mathrm{c} \quad 1.25 \quad 4.00$ $\begin{array}{llllll}\text { RED CHILI _. } & 10 \mathrm{c} & 50 \mathrm{c} & 1.60 & 5.00\end{array}$ MAMMOTH VIRGINIA PEANUTS 1 lb. 30 cts. 5 lbs. 1.25 GARDEN PEAS

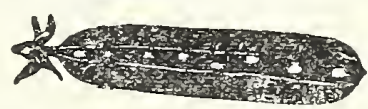

Early Alaska (About 36 inches high) $1 \mathrm{lb} .30$ cts. 2 lbs. 55 cts. 5 lbs. 1.25 Early May or First \& Best (36 inches high) $1 \mathrm{lb} .30 \mathrm{cts} 2 \mathrm{lbs} .55 \mathrm{cts} \quad 5$ lbs. 1.25 Little Marvel (about 15 inches high)

$1 \mathrm{lb} .50 \mathrm{cts}$. $2 \mathrm{lbs} .95 \mathrm{cts} .5 \mathrm{lbs} .2 .00$ Gradus or Prosperity (About 30 inches high) $1 \mathrm{lb} .40 \mathrm{cts} .2 \mathrm{lbs} .75 \mathrm{cts} .5 \mathrm{lbs} .175$

Thomas Laxion (About 30 inches high)

$1 \mathrm{lb} .40 \mathrm{cts} . \quad 2 \mathrm{lbs} .75 \mathrm{cts} . \quad 5$ lbs. 1.75 Telephone (About five feet high)

1 lb. 45 cts. 2 lbs. 85 cts. 5 lbs. 2.00 Champion of England (Five feet high)

$1 \mathrm{lb} .40 \mathrm{cts}$. $2 \mathrm{lbs} .75 \mathrm{cts}$. 5 lbs. 1.75

1 lb. Black Eye

5 lbs. 1.25

PUMPKINS

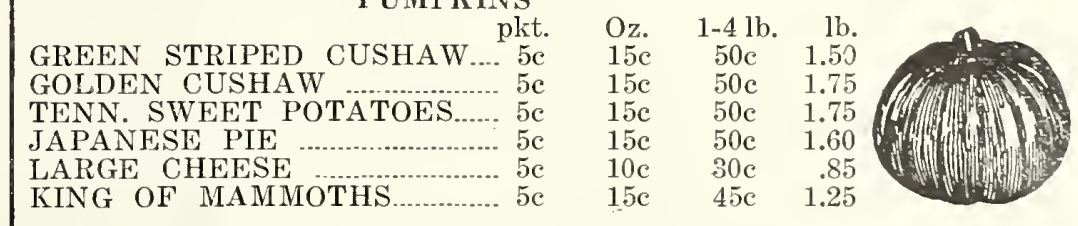

RADISHES

CINCINNATI MARKET pkt. Oz. 1-4 lb. lb. CRIMSON GIANT ……...... 5 c $10 \mathrm{c} \quad 30 \mathrm{c} \quad 1.00$ EARLY SCARLET TUKNII'..... $5 \mathrm{c}$ 10c $30 \mathrm{c} \quad .90$ WH. TIP SCARLET TURNIP .... $5 \mathrm{c}$ 10 e $30 \mathrm{c} \quad .95$ LONG SCARLET SHORT TOP.. 5c $10 \mathrm{c}$ 30c 1.00 FRENCH BREAKFAST ........... 5c $5 \mathrm{c}$ 10 WHITE STRASSBURG $\ldots . . . . . . . . . . .5 \mathrm{c} \quad 10 \mathrm{c} \quad 30 \mathrm{c} \quad .90$ WHITE ICICLE …..................... $5 \mathrm{c} \quad 10 \mathrm{c} \quad 30 \mathrm{c} \quad 1.00$

RHUBARB

LINNAEUS 
